\title{
ASPECTOS SOCIALES Y CULTURALES DE LA EDUCACIÓN MATEMÁTICA
}

\author{
BISHOP, A.J. \\ Department of Education. University of Cambridge.
}

Conferencia invitada en el Il Congreso Internacional sobre Investigación en la Didáctica de las Ciencias y las Matemáticas. Valencia 23-25 de septiembre de 1987.

(Traducción de Enrique Vidal).

\section{SUMMARY}

This paper deals with two fieids of research which have contributed to revise the teaching of Mathematics over the last decade: those concerned with social and cultural aspects of Mathernatics education.

Voy a hablar de dos áreas de investigación en desarrollo dentro de la didáctica de las matemáticas. Ambas han estado presentes en nuestros planteamientos anteriores, pero se han convertido en áreas de estudio significativas durante los diez últimos años aproximadamente.

Admito que diez años es un abrir y cerrar de ojos según la escala temporal de la historia de España, pero ha sido el tiempo suficiente para que Ileguemos al convcncimiento de que ahora podemos crear, para nucstros alumnos, una educación matemática mejor que la que tuvimos antes. Sucede esto porque esas dos áreas reconocen que lo fundamental de toda cducación son las personas y la educación matemática no es una excepción.

Puede parecer una observación extraña, pero en nuestro mundo, cada día más tecnológico, la cducación ha sido desarrollada para servir a las necesidades de la tecnología, bien de una manera directa, bien indirectamente a través de estructuras sociales creadas para poten. ciarla. La educación matemática ha sido, por supuesto, muy importante en este desarrollo, ya que la ciencia y la tecnología dependen de las ideas matemáticas. Así, la enseñanza de las matemáticas se ha ido gradualmente convirtiendo más y más en preparación matemática, insistiendo en instruir a los alumnnos en cómo, mediante métodos metemáticos correctos, obtener resultados adecuados.

También ha resultado una preparación muy selectiva, $y$, asi, sólo aquellos estudiantes que tienen la habilidad de obtener muchas respuestas correctas pueden seguir hacia matemáticas más elevadas.
Pero, ¿es este proceso de aprender más y más técnicas lo que le da a nuestros alumnos una adecuada formación? ¿Están matemáticamente formados como resultado de esta preparación matemática eficiente? No lo creo. Lo que agrava todo esto es que esta preparación matemática no es tan eficiente como a veces se podría pensar. En la mayoría de los países, y supongo que España no es diferente, muchos estudiantes fracasan al aprender técnicas matemáticas, al intentar obtener resultados correctos y, en definitiva, en sus cursos de matemáticas.

En todos los países, los estudiantes tienen fracasos en sus clases de matemáticas $\mathrm{y}$, como consecuencia, se encuentran muy desanimados con esta materia. Existe tal animadversión que si yo le digo a alguien que soy un profesor de matemáticas me mira como si fuera un bicho raro. Si le digo que me gustan las matemáticas, piensa que estoy loco. Si comento que puedo ayudar a que también a los demás le gusten, simplemente no me creen. En general de las matemáticas se piensa que no son para ser estudiadas o para disfrutar de ellas, sino más bien para ser sufridas como una tortura necesaria para la mente.

Podríamos pensar como consecuencia que este tipo de personas quieren suprimir las matemáticas del currículo escolar. «Si todo el mundo las odia tanto, librémonos de ellas». ¡Pues no! «Son importantes», dicen. "Debes esdudiarlas», les insisten a sus hijos. "Conseguirás un buen trabajo, quizá, si las estudias bien” ¿Quizá sí? O quizá no.

Asi pues, ¿cuál es la solución de esta paradoja? Claramente, las matemáticas son importantes en nuestro cu- 
rrículo escolar. Lo es en el currículo de todos los países, y no van a estar equivocados millones de personas. Las matemáticas no son tan malas en si mismas. Son, simplemente, parte del conocimiento humano. Debe de ser nuestra enseffanza la que está todavía subdesarrollada. Necesitamos reexaminarnos y desarrollar las vías de enser̃anza de las matemáticas a nuestros escolares. Es aqui donde surge la investigación, con las siguientes finalidades: ayudar a reexaminar la enseñanza de las matemáticas, desarrollar nuevos caminos pa. ra pensar sobre este proceso, y formar y preparar más gente en esas nuevas vías de pensamiento.

Las dos áreas de investigación en desarrollo que hoy voy a tratar son: primeramente la referida a los aspectos sociales, y en segundo lugar la relativa a los culturales. Por supuesto que están relacionadas y, en particular, ambas tratan de las personas involucradas directa o indirectamente en la educación matemática.

La dimensión social de la educación matemática es evidente a diferentes escalas. Es un campo nuevo y fascinante, que interesa a profesores e investigadores. Vamos a tratar brevemente cada una de esas escalas y a describir algo de lo que se está haciendo en ellas.

Primeramente, a escala individual, nos encontramos con investigaciones que demuestran cómo el aprendizaje del individuo está influenciado por el aprendizaje de los demás (Hoyles, 1982; Webb, 1982; Bishop, 1985). Todos sabemos por nuestra experiencia lo importantes que son los otros estudiantes, y cualquier profesor conoce la interacción entre sus ahumnos. Sólo necesitamos observar una clase 10 ó 15 minutos, para que, tápidamente, nos enteremos de quién influencia a quién; quiénes son las personalidades dominantes y quiénes los «seguidores» (¿las ovejas?).

Cualquier buen profesor conoce y usa esta información en su enseñanza, pero sólo ahora hemos empeza-

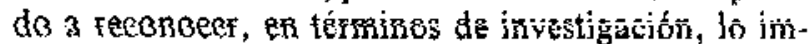
portante que es. Parece que es particularmente significativo en relación con los sentimientos, opiniones, actitudes y aspectos afectivos en general.

Esto no significa que los aspectos cognitivos no estén también muy implicados en el proceso. El grupo de investigación de Génova (Perret-Clermont y SchubanerLeoni, 1981) es muy consciente de los aspectos interpersonales en el desarrollo cognitivo de los escolares. Basta con escuchar a un grupito de niños ocupado en «enseñarse» unos a otros a la salida del colegio. ¿Puede realmente pensarse que los escolares no se ayudan mutuamente en su desarrollo cognitivo?

En una segunda cscala, la clase como grupo de personas presenta aspectos sociales significativos. El papel de maestros y profesores es muy importante desde esta perspectiva. Muchos estudiantes nos muestran cómo los puntos de vista del profesor se transmiten tanto a sus alumnos, que terminan pensando según sus directrices. Por ejemplo, las chicas aprenden que ellas no serán tan buenas en matemáticas como los chicos, los que son más lentos en el aprendizaje se sienten inferiores y los más rápidos superiores (Becker, 1982; Lorenz, 1982). En inglés se denomina esto «self-fulfilling prophesy». Los alumnos se estimulan, o se desalientan, según las expectativas que sus profesores tienen de ellos.

Sin embargo, no culpemos a los profesores. Si han de enseñar a gnupos numerosos, inevitablemente tendrán problemas. Si no tienen tiempo de desarrollar la personalidad de cada alumno, de alguna forma han de realizar su trabajo. Si no pueden conocer a fondo a cada uno, tendrán que manejar estereotipos de alumnos.

Lo que nos indican estas investigaciones es que necesitamos usar más métodos de enseñanza de grupos reducidos. Debemos dejar que los alumnos, bajo el control del profesor, se formen unos a otros, dialoguen más, trabajen en proyectos de grupo, y adquieran más responsabilidad en el desarrollo de su propio conocimiento. $\mathrm{Si}$ los profesores pueden formar buenos grupos pequeños para realizar actividades, notarán que tienen más tiempo para dedicarlo a los alumnos que realmente lo necesitan.

En la siguiente escala social, la del centro o institución de enseñanza, la investigación nos muestra otros aspectos que tienen efectos significativos en la educacion matemática de los estudiantes. En este nivel lo más importante son las relaciones entre los profesores, y entre éstos y los demás responsables del centro.

Empieza a estar claro que las matemáticas escolares, la materia con la que se encuentran los alumnos, depende de los puntos de vista e ideas de mucha gente, $y$, por ejemplo, en todo el Reino Unido los centros de enseñanza secundaria se organizan en departamentos, uno de los cuales es el de matemáticas.

Fisie depariamento está formado por todos los proficsores que enseñan matemáticas, con un jefe de dcpartamento; se dispone de un lugar que sirva como centro de recursos y despacho, realizándose reuniones regulares. (Bishop and Nickson, 1983).

Esto significa que todos los responsables de enseñar matemáticas pueden discutir y llegar a estar de acuerdo sobre sus ideas y métodos, tienen la posibilidad de ayudarse unos a otros, y pueden conseguir conjuntamente que, por ejemplo, sus horarios sean adecuados a las necesidades de la materia en el currículo escolar. Esos colectivos sirven tanto para satisfacer las necesidades de relación, como de organización.

En la cuarta escala social nos movemos fuera de la institución educativa, dentro de lo que es la sociedad en su sentido más amplio. Ciertamente no está claro que la educación matemática en el Reino Unido tenga que ser la misma que en los Estados Unidos, Francia o España. Cada país tiene sus propios planes politicos, económicos y sociales, y pueden ser útiles para nosotros 
los estudios e investigaciones sobre sus diferencias y semejanzas. The Second International Mathematical Study está publicando periódicamente sus hallazgos y estamos aprendiendo mucho de su gran cantidad de datos. También en este sentido, un libro que es muy interesante (Swetz, 1978) tiene por título Socialist Mathematics Education, y trata de la educación matemática en varios países con un sistema político socialista.

Todo esto significa que no debemos transferir ideas ciegamente de un pais a otro. Hemos de tener en cuenta nuestras diferencias y sus implicaciones. En el Reino Unido, por ejemplo, desde los 14 años, incluso aunque haya una gran mezcla de alumnos en los centros, las clases se hacen más y más selectivas. Sólo tenemos un pequeño porcentaje de nuestros alumnos que llega a la Universidad, y ese pequeño grupo está gradualmente seleccionado desde los 14 años. Algunos afirman que incluso empieza antes.

A mí no me gusta eso y creo que no es lo adecuado. Pero, mientras yo no estoy de acuerdo con muchas cosas inuestro Gobierno sigue manejando nuestro Sistema Educativo! Así pues, trato de usar mi influencia para cambiar ambas cosas: ¿EI Gobierno y el Sistema Educativo!

No hay duda, por tanto, de que la politica juega un papel importante en la determinación del tipo y la calidad de la enseñanza de las matemáticas. Si ustedes quieren verla progresar en su país, es importante que comprendan las mutuas influencias de las diferentes partes del entramado educativo. Haciendo uso de otro ejemplo, es interesante señalar cómo en los Estados Unidos los hallazgos de la investigación son llevados a los libros de texto para mejorarlos, con más frecuencia que en el Reino Unido. Nosotros también intentamos mejorar la calidad de la enseñanza, pero preferimos centrarnos en una mejor preparación de maestros y profesores. Esto podría significar que quizás nuestros libros de texto son peores y que, en cambio, alentamos a los profesores a que ellos mismos desarrollen sus propios materiales de enseñanza.

Hemos tratado brevemente los aspectos sociales en cuatro niveles, el individual, la clase, el centro y la sociedad. La quinta escala nos lleva a la cultura, que es la otra importante área general a la que quiero referirme.

Hace más o menos cinco años el criterio general mantenía que las matemáticas eran un conocimiento independiente del entorno cultural. Después de todo, se argumentaba "menos por menos es igual a más», en todas partes, y los triángulos en cualquier lugar del mundo tienen ángulos que suman 180 grados en total. Sin embargo este punto de vista confundia la «universalidad de la verdad" de las ideas matemáticas, con la base cultural de este conocimiento. De acuerdo en que esas afirmaciones son ciertas en cualquier parte del mundo, ya que las ideas se descontextualizan y abstraen de manera tal que «obviamente» se pueden aplicar en cualquier sitio. Pero, ¿de dónde surgen los números negativos? ¿Por qué los ángulos de un triángulo suman 180 grados y no, por ejemplo, 100? Porque las ideas matemáticas tienen una historia cultural. Ese es el porqué. Las ideas religiosas no son diferentes y, desde luego, podemos fácilmente reconocer distintas religiones en diferentes culturas. ¿Podremos también reconocer la existencia de diferentes matemáticas?

Recientemente se ha aclarado de forma convincente, a partir de investigaciones antropologicas y estudios comparativos de diferentes culturas, que las matemáticas que nosotros conocemos son un hecho cultural, y que otros grupos culturales han creado ideas que claramente son «otras matemáticas». Podemos citar el trabajo de Zaslavsky (1973), quien ha señalado en su Iibro Africa Counts el alcance de las ideas matemáticas en las culturas indígenas africanas. Otra fuente de información es Gerdes (1985). En otros continentes, las investigaciones de Lancry (1983), Lean (1986) y Bishop (1979) en Papúa Nueva Guinea, Harris (1980) y Lewis (1976) en la Australia aborigen y Pinxten (1983) y Closs (1986) con indios americanos, nos han mostrado también hechos evidentes, que nos llevan a afirmar como conclusión que la matemática es una actividad pan-humana. Es decir, que todos los grupos culturales desarrollan matemáticas, igual que todos desarroIlan lenguaje, religión, juegos y arte.

Además, todos los grupos culturales desarrollan sus propios lenguajes, religiones, etc. De la misma forma lo hacen con sus propias matemáticas. Así pues podemos reconocer la existencia de diferentes matemáticas.

Este tipo de investigación está siendo muy estimulado por el trabajo realizado en muchos países, incluyendo el Reino Unido, acerca de la educación de niños cuya cultura familiar no coincide con la del colegio. Como cuestión particular, se estudia la relación entre la cultura familiar del niño y el currículo escolar de matemáticas.

En los centros escolares de muchos paises, el currículo escolar refleja, como consecuencia de determinadas presiones, la naturaleza multicultural de sus sociedades, $y$ ha sido generalmente reconocida la necesidad de reevaluar la experiencia académica en su conjunto, teniendo en cuenta el fracaso educativo de muchos ninos que proceden de comunidades étnicas minoritarias. En algunos países como Papúa Nueva Guinea, Mozambique e Irán, hay acuerdos para reexaminar la experiencia educativa, "colonial» u "occidental», y tratar de crear en su lugar, una educación que esté a tono con la cultura "de casa» de esas sociedades. Los mismos acuerdos surgen en otros debates acerca de la educacion de minorias australianas, de indios americanos, de los lapones o de los esquimales. En todos esos casos, una situación de conflicto cultural está reconocida y los currículos están siendo reexaminados. 
Los currículos de matemáticas, sin embargo, han cambiado lentamente, debido en parte al malentendido descrito anteriormente.

Es actualmente una necesidad urgente encontrar los caminos del curriculo de matemáticas «multicultural». La dificultad se centra en el hecho de que las matemáticas en el curriculo escolar no han sido hasta ahora consideradas como un hecho cultural, y por ello, para ir hacia un «multiculturalismo» debemos tratar primero de "culturizarlas».

Éste es el problema en el que yo he estado trabajando durante los últimos años y me gustaria contarles brevemente lo que he encontrado.

Básica y brevemente, las matemáticas pueden entenderse como una cierta tecnología simbólica, algo parecido a un lenguaje. (No como un lenguaje, sino $p a-$ recido a un lenguaje - similar, pero diferente).

Resulta de seis tipos de actividades relacionadas con el entorno, en las que todos los grupos culturales participan y que por lo tanto son universales. Estas actividades son:
contar
localizar
medir
diseñar
jugar
explicar

Cada una de ellas desarrolla ideas importantes para nuestras matemáticas.

Contar desarrolla Numeros. Nombres para los números. Pautas. Bases. Sistemas numéricos. Cuantificadores. Magnitud discreta.

Localizar Dimensiones. Coordenadas. Ejes. Caminos. Redes. Simetría. Topologia. Distancia y dirección. Lugares geométricos.

Medir Orden. Tamaño. Unidades. Sistemas de medida. Precisión. Magnitud continua.

Diseñar Forma. Regularidad. Pautas. Construcciones. Dibujo. Representación. Geometria.
Jugar

Explicar

Reglas. Procedimientos. Planes. Modelo. Juego. Satisfacción. Competición. Cooperación.

Clasificación. Convenciones. Argumentos. Lógica. Prueba. Relato. Conectivas.

Asi pues, desde el punto de vista educativo, podemos empezar a pensar en la educación matemática como un posicionamiento de los alumnos en una parte de su cultura. (Acabo de terminar un libro titulado Mathematical Enculturation, que analiza ese planteamiento, y que aparecerá en 1988). En este contexto, el otro problema educativo importante es el mal emparejamiento entre la cultura del alumno y la de la sociedad en general, como ya señalé anteriormente. Afortunadamente, analizando la estructura cultural de las ideas matemáticas, tal como acabamos de hacer, podemos empezar a utilizar este entramado de seis actividades como una estructura cultural útil para el curriculo. Esto significa que es muy posible remodelar el currículo escolar de matemáticas en términos de las seis actividades y así hacer posible a los profesores de matemáticas de cualquier lugar relacionar una cultura particular de determinados niños con otra cultura. Esto es lo que empezamos ahora a hacer en el Reino Unido y las posibilitades son enormes.

No tenemos más tiempo para entrar en detalles. Lo único que podemos hacer es tratar de sintetizar y terminar diciendo que nunca debemos olvidar que la educación matemática, como cualquier otra educación, trata de personas.

El conocimiento social y cultural que se está desarroIlando por las investigaciontes en todo el mundo refleja un creciente reconocimiento de este hecho. Desde mi punto de vista, es un desarrollo muy alentador y algo que me alegra defender.

Para mí es el único camino por el que podemos crear para nuestros jovenes estudiantes una auténtica edicación matemática. Confio en que nos ayudará a que las matemáticas sean menos odiadas, se entienda mejor en qué consisten, como sirven para comprender nuestro mundo, y por qué son tan importantes para los escolares.

\section{REFERENCIAS BIBLIOGRÁFICAS}

BECKER, J.R., 1982, Differential treatment of females and males in mathematics classes, Journal for Research in Mathematics Education, 12, 1, 40-53.
BISHOP, A.J., 1979, Visualising and Mathematics in a PreTechnological Culture, Educational Studies in Mathematics, 10, 2, 135-146. 
BISHOP, A.J., 1985, The Social Psychology of Mathematics Education, in L. Streefland (ed.), Proceedings of the Ninth International Conference for the Psychology of Mathematics Education. (Noordwijkerhout, Holland).

BISHOP, A.J. 1988, Mathematical Enculturation - A Cultural Perspective on Mathematics Education, in press. (Reidel: Holland).

BISHOP, A.J. and NICKSON, M., 1983, Research on the Social Context of Mathematics Education. (NFERNelson: Slough).

CLOSS, M.P. (ed.), 1986, Native American Mathematics. (University of Texas Press, Austin: Texas).

GERDES, P., 1985, Conditions and Strategies for Emancipatory Mathematics Education in Undeveloped Countries, For the Learning of Mathematics, 5,1, 15-20.

HARRIS, P., 1908, Measurement in Tribal Aboriginal Communities. (Northern Territory Department of Education: Australia).

HOYLES, C., 1982, The pupil's view of Mathematics learning, Educational Studies in Mathematics, 13, 349-372.

LEAN, G.A., 1986, Counting Systems of Papua New Guinea, Research Bibliography, 3rd edn. (Department of Mathematics, Papua New Guinea University of Technology,
Lae: Papua New Guinea).

LEWIS, D., 1976, Observations on route-finding and spatial orientation among the Aboriginal peoples of the Western desert region of central Australia, Oceania, XLVI, 4, 249-282.

LORENZ, J.H., 1982, On some psychological aspects of mathematics achievement and classroom interaction, Educa tional Studies in Mathematics, 13, 1-19.

PERRET-CLERMONT, A.N. and SCHUBAUER-LEONI, M.L., 1981, Conflict and cooperation as opportunities for learning in P. Robinson (ed.) Communication in Development. (Academic Press: New York).

PINXTEN, R., VAN DOOREN, I, and HARVEX, F., 1983, The Anthropology of Space. (University of Pennsylvania Press).

SWETZ, F.J. (ed.), 1978, Socialist Mathematics Education. (Burgundy Press, Southampton, P.A.)

WEBB, N., 1982, Group composition, group interaction and achievement in cooperativee small groups, Journol of Educational Psychology, 74, 4, 475-484.

ZASLAVSKY, C., 1973, Africa Counts. (Prindle, Weber and Schmidt, Inc.: Boston, Mass). 